\title{
Spanish Translations of Selected Abstracts
}

\author{
Translated by Graciela Zapata, Sergio San Martín, and Silvia Lorena Zapata
}

Modificaciones Recomendadas y Aplicación del Sistema de Comando de Incidentes Hospitalario para el manejo de Emergencias en Hospitales Hoy

\section{Resumen}

El Sistema de Comando de Incidentes Hospitalario (HEICS), el cual se encuentra en su tercera edición, ha surgido como un modelo popular de sistema de comando de incidentes para la respuesta a emergencias en hospitales de los Estados Unidos y otros países. Desde su creación en 1991, varios eventos en los años recientes, han transformado los requerimientos del manejo de emergencias hospitalarias, incluyendo los ataques con sarin al metro de Tokio en 1995, los ataques postales con ántrax en los Estados Unidos en el 2001 y el surgimiento del SARS en Asia del este y Toronto en el 2003.

Se han sugerido varias modificaciones al HEICS, para dar cumplimiento a las necesidades actuales del manejo de emergencias hospitalarias, incluyendo: (1) un Consultor de Incidente en la sección administrativa del HEICS, para proporcionar asesoria de experto al Comandante de Incidente en emergencias químicas, biológicas, radiactivas y nucleares (CBRN), según sea necesario; así como asesoría en cuestiones de salud mental; (2) nuevos líderes de unidades en la Sección de Operaciones para coordinar el manejo de pacientes contaminados o infecciosos en las emergencias CBRN antes mencionadas; (3) nuevos líderes de unidad en la Sección de Operaciones para coordinar el apoyo emocional (salud mental) para pacientes, visitantes, miembros del equipo de salud, voluntarios y demás personas involucradas en emergencias relacionadas con terrorismo o eventos que produzcan la necesidad de brindar apoyo psicológico; (4) un nuevo Líder de la Unidad de Descendientes/Expectantes para coordinar el manejo de ambos tipos de pacientes integralmente; y (5) un nuevo Líder de la Unidad de Tecnología de Información en la Sección de Logística para coordinar el manejo de la tecnología de información y sistemas.

También se recomiendan nuevos usos del HEICS, en el manejo de emergencias hospitalarias, incluyendo: (1) la adopción del HEICS como marco conceptual para organizar todas las fases del manejo de emergencias hospitalarias, incluyendo mitigación, preparación, respuesta y recuperación; y (2) la aplicación del HEICS no sólo a las instalaciones de los centros hospitalarios, sino también a los sistemas de salud en general.

Finalmente se sugieren tres niveles de competencia para los trabajadores del equipo de salud en el HEICS: (1) entendimiento básico del HEICS para todos los trabajadores del equipo de salud, (2) entendimiento avanzado y desarrollo de habilidades en el HEICS para trabajadores que podrian asumir puestos de liderazgo en la respuesta a emergencias hospitalarias y (3) desarrollo especial de habilidades en la implantación y adecuación del HEICS, para los trabajadores del equipo de salud en ambientes con pocos recursos. El HEICS debe ser visto como un trabajo que madura conforme surgen nuevos retos y mientras los hospitales obtienen mayor experiencia en su uso.

Arnold JL, Dembry LM, Tsai MC, Dainiak N, Rodoplu U, Schonfeld D, Parwani V, Paturas J, Cannon C, Selig S: Modificaciones Recomendadas y Aplicación del Sistema de Comando de Incidentes Hospitalario para el manejo de Emergencias en Hospitales Hoy. Prebosp Disast Med 2005;20(5):290-300.

Modelación de Recursos para Desastres: Medicamentos requeridos para un Equipo de Respuesta a Desastres

\section{Extracto}

Introducción: La respuesta a un desastre involucra muchos factores además del personal, como el equipo medico, el no medico y los suministros. En particular, cuando los equipos para desastres responden, deben hacerlo con suficientes suministros y equipo para manejar a los pacientes esperados por muchos días antes del reabastecimiento. Con el fin de que este proceso sea eficiente y expedito, es necesaria una precisa y previa planeación, de los suministros necesarios para los trabajadores del desastre. Estos suministros deben de ser proveídos, tanto para el cuidado medico general, así como para los problemas específicos de los desastres.

Problema: El objetivo fue desarrollar un modelo que provea un marco de trabajo, para la determinación de las necesidades de suministros para el Sistema Medico Nacional para Desastres, los Equipos de Atención Medica en Desastres u otros equipos de respuesta en desastres, en un ambiente civil

Métodos: Se modelo un hospital comunitario, para determinar las características de los pacientes ingresados al departamento de urgencias, incluyendo los datos demográficos de los pacientes, sus principales padecimientos, medicamentos administrados durante su estancia y los recetados al darlos de alta, así como los exámenes de laboratorio utilizados, para investigar los requerimientos de suministros de un equipo de desastres. La información fue capturada en un programa de seguimiento de pacientes, así como recolectada de varios sistemas hospitalarios de recolección de información. La información del hospital comunitario, fue comparada contra la información publicada, por miembros del Sistema 
Medico Nacional para Desastres, de dos desastres previos por huracán.

Resultados: Hasta el punto posible, el modelo predijo la proporción de padecimientos de los pacientes y por lo tanto, el tipo de cuidado y suministros necesarios para el manejo de los pacientes.

Conclusión: Proponemos que este modelo, es el primer paso en la preparación para el despliegue de los equipos médicos para desastres

Rosenthal MS, Klein K, Cowling K, Grzybowski M, Dunne R: Modelación de Recursos para Desastres: Medicamentos requeridos para un Equipo de Respuesta a Desastres. Prebosp Disast Med 2005;20(5):309-315.

Tiopental vs. Etomidato para la Secuencia de Intubación Rápida (SIR) en Medicina Aérea

\section{Resumen}

Introducción: Aunque existe un acuerdo general en el que se establece que la secuencia de intubación rápida (SIR), es la técnica preferida para la intubación del paciente en medicina de aviación, se han usado varias alternativas sin una evidencia clara, que indique cual de ellos es superior.

Hipótesis: El presente estudio, se diseño para comparar el uso del etomidato (ETOM) contra el del tiopental (THIO) como agentes adjuntos usados conjuntamente con succinilcolina ( $\mathrm{SCh}$ ) para una secuencia de intubación rápida en un sistema aeromédico urbano.

Métodos: Se realizó un estudio retrospectivo (antesdespués) utilizando la metodología de revisión de gráficas con la asistencia de una computadora. Los pacientes adultos de un periodo de 2 años que recibieron THIO para secuencias de intubación rápida se compararon con los que recibieron ETOM en un periodo similar después de un cambio de protocolo en el que se indicaba el ETHOM en lugar del THIO para todas las intubaciones.

Resultados: No se encontraron diferencias en ninguno de los puntos finales primarios. El tiempo de estabilización (13.1 vs. 12.9), el número de intentos de intubación (1.1 vs. $1.2)$, los intubaciones exitosas al primer intento $(90 \%$ vs. $82 \%)$, las intubaciones exitosas globales ( $100 \%$ vs. $96 \%$ ) y el tiempo de intubación (18.4 vs. 21.7) fueron similares para todas las comparaciones de THIO contra ETOM (en todos los casos $p>0.05$ ).
Conclusión: No se encontraron diferencias clínicamente relevantes entre el etomidato y el tiopental al ser utilizados conjuntamente con la succinilcolina en intubaciones realizadas en medicina de aviación.

Sonday CH, Axelband J, Jacoby J, Higgins R, Crider D: Tiopental vs. Etomidato para la Secuencia de Intubación Rápida (SIR) en Medicina Aérea. Prehosp Disast Med 2005;20(5):324-326.

Creación de Puntos de Referencia para la evacuación hospitalaria: Recolección Crítica de Datos

\section{Resumen}

En eventos como los terremotos o ataques terroristas, los hospitales pueden convertirse en víctimas del desastre. Es posible que surja la necesidad de transferir pacientes a instalaciones externas, en lugar de seguir proporcionándoles tratamiento localmente. Posteriormente al terremoto de Northridge, ocho hospitales en el área afectada fueron el foco de un estudio por parte de la Fundación Nacional de Ciencia, que evaluó el status de la planeación previa al evento de cada hospital, la decisión de evacuar posterior al evento y los procesos de evacuación internos y externos. Basados en estas experiencias, el documento ofrece una herramienta de recolección de datos estandarizada que permitirá a los investigadores, registrar la información de una evacuación hospitalaria de una forma sistemática, de manera que se pueda obtener datos comparables, mejorar la metodología de investigación sobre evacuaciones y generar consenso en los métodos. El objetivo principal del estudio incluye la demografía del hospital, la descripción de los planes existentes de respuesta a desastres, el impacto del evento en las operaciones del hospital, la toma de decisiones, el comando de incidentes, el desplazamiento de pacientes dentro de las instalaciones y hacia instituciones externas, así como la recuperación del hospital.

Schultz CH, Koenig KL, Auf der Heide E: Creación de Puntos de Referencia para la evacuación hospitalaria: Recolección Crítica de Datos. Prehosp Disast Med 2005;20(5):331-342. 